\title{
10-Dimensional Cosmology With Gauss-Bonnet Gravity In Generalized Scalar-Tensor Theories From Superstring Theories
}

\author{
EL-NABULSI Ahmad Rami* \\ Department of Nuclear Engineering, Cheju National University, Ara-dong 1, Jeju 690-756, South Korea
}

(Received on 24 August, 2009)

\begin{abstract}
Superstring theories are promising candidates for unifying gravity with all matter interactions. In this work, we discussed a particular 10-dimensional cosmology based on Gauss-Bonnet invariant term coupled to a generalized scalar-tensor theory as resulting from superstring theories. Some interesting features concerning the late-time dynamics are explored and described in some details.
\end{abstract}

Keywords: Gauss-Bonnet gravity, higher-dimensions, generalized scalar-tensor theories, dark energy.

One of the most recent surprising discoveries in modern cosmology is the realization that our universe is accelerated in time and is dominated by a mysterious dark energy which accounts for about $70 \%$ of the total energy content. The remaining $30 \%$ contribution is largely due to dark matter. These data is based on the accumulations of recent astronomical events in particular the Hubble diagram of supernovae type Ia, [1,2] cosmic microwave background (CMB) anisotropies [3], the large scale galaxies structures of the universe [4] and Sachs-Wolfe effects [5]. This is strong evidence in favour of the existence of a dark energy (DE) fluid which may be the realization of a positive cosmological constant which is considered as the first natural theoretical explanation of the accelerated expansion of the cosmos.

Many leading candidates for the dark energy were discussed largely in literature including the K-essence with modified kinetic energy [6], viscous fluid [7], Chaplygin gas [8,9], Generalized Chaplygin gas model (GCGM) [10,11], Brans-Dicke (BD) pressureless solutions $[12,13,14]$, decaying Higgs fields [15], dilaton field of string theories with gaugino condensation [16], tachyon (unstable field) as a dark energy source, $[17,18]$ etc. Some other nice alternatives scalar theories include the stringinspired dilaton gravities and the higher derivative theories with an additional quadratic scalar curvature [19], GaussBonnet (GB) cosmology [20], etc. These modified theories of gravity revealed interesting consequences and have potential to provide a linkage between the accelerated expansion of the universe and fundamental physics although the acceleration cannot be explained by the standard model of particle physics and classical general relativity.

Recently, a great deal of interest has been done in extradimensional cosmology as it may provides a possible understanding the smallness of the cosmological constant problem and a solution to the hierarchy problem. [21] From the point of view of string theory, the GB term may be said to be preferred, as they lead to a unitary and low energy effective theory free from ghosts. [22] In this work, we will look at ten-dimensional late-time cosmology with Lovelock gravity non-minimally coupled to the scalar field. The nonminimal coupling is in fact required in many quintessence models based on supergravity arguments as well as quantum corrections to the scalar field theory and its renormalizabil-

*Electronic address: nabulsiahmadrami@yahoo.fr ity in curved spacetime. Moreover, in induced gravity theory (quantum field renormalization theory) gravity results from nonminimally scalar field coupling; other sources are also the superunification theories (superstring and Kaluza-Klein theories, wormholes, cyclic universe, etc.). [23-30] This term also plays a primary role in many alternatives frameworks including the Chern-Simons gravitational theories and the renormalization of quantum field theories in curved spacetime. [31] The most remarkable feature of the GB gravity is that at high energy limit it drastically transforms radial dependence from inverse to proportionality as singularity is approached and thereby making it weak. [32] In other words, all solutions approach de Sitter spacetime at high curvature, which is naturally singularity-free.

The action of the theory in ten-dimension is [26]

$$
\begin{aligned}
S & =\int d^{10} x \sqrt{-g_{10}} \\
& \times\left[f(\phi) R-\phi^{-1} \omega(\phi) \phi_{, A} \phi^{, A}-V(\phi)-\kappa^{2} L_{m}+C G(R)\right] .
\end{aligned}
$$

Here

a) $R$ is the scalar curvature,

b) $\omega(\phi)$ is the Brans-Dicke coupling function between the dynamical scalar field and the gravity,

c) $f(\phi)=\phi$ is the coupling scalar function with the Ricci scalar, i.e. geometry

d) $V(\phi)$ is the potential function playing the role of an effective cosmological constant of the theory,

e) $g_{10}$ is the 10 -dimensional scalar metric,

f) $G(R)=R^{2}-4 R_{A B} R^{A B}+R_{A B C D} R^{A B C D}$ is the quadratic GB term,

g) $C$ is the Gauss-Bonnet coefficient

h) $\kappa^{2}$ is the gravitational coupling constant set equal to one here for mathematical simplicity,

i) $L_{m}$ represents the matter-Lagrangian,

j) the indices run $A, B, \ldots \in(0, \ldots, 9)$. 
The field equations that followed from the action (1) are:

$$
\begin{aligned}
& f\left[R_{A B}-\frac{1}{2} g_{A B} R\right]+\frac{1}{2} g_{A B}\left[\frac{\omega(\phi)}{\phi} \phi_{, E} \phi^{, E}+V(\phi)\right] \\
& -\frac{\omega(\phi)}{\phi} \phi_{, A} \phi_{, B}-f_{; A ; B}+f_{; A}^{; A} g_{A B} \\
& +\frac{C}{2}\left[R_{A B C D} R^{A B C D}-4 R_{A B} R^{A B}+R^{2}\right] g_{A B} \\
& -C\left[2 R_{A C D E} R_{B}^{C D E}+2 R R_{A B}-4 R_{A B C D} R^{C D}-4 R_{A C} R_{B}^{C}\right] \\
& =\frac{1}{2} T_{A B}, \\
R f^{\prime} & +2 \frac{\omega(\phi)}{\phi} \phi_{; A}^{; A}+\left(\frac{\omega^{\prime}(\phi)}{\phi}-\frac{\omega(\phi)}{\phi^{2}}\right) \phi^{, A} \phi_{, A}-V^{\prime}(\phi)=0
\end{aligned}
$$

where $f_{; A}^{; A} \equiv f^{\prime \prime} \phi^{, A} \phi_{, A}+f^{\prime} \phi_{; A}^{; A}$ and $f^{\prime} \equiv d f / d \phi, f^{\prime \prime}=$ $d^{2} f / d \phi^{2}$. The energy-momentum tensor in theory is

$$
T_{B}^{A}=\operatorname{diag}(-\rho, p, p, p, P, P, P, P, P, P),
$$

where $\rho, p$ and $P$ are the matter density, visible pressure and extra-dimensional pressure respectively.

The metric we adopt in our theory corresponds to a flat and homogeneous spacetime and looks like: [26,33]

$$
d s^{2}=\underbrace{-d t^{2}+a^{2}(t) \sum_{i=1}^{3} d x^{i} d x^{i}+}_{\text {visible FRW }}+\underbrace{b^{2}(t) \sum_{j=1}^{6} d y^{j} d y^{j}}_{\text {extra-dimensions }} .
$$

With these considerations the Friedmann field equation and equation (3) are given by:

$$
\begin{aligned}
& 3 H_{a}^{2}+18 H_{a} H_{b}+15 H_{b}^{2}+36 C H_{b} \\
& \times\left(2 H_{a}^{3}+15 H_{a}^{2} H_{b}+20 H_{a} H_{b}^{2}+5 H_{b}^{3}\right) \\
= & V(\phi)+\frac{8 \pi \rho}{\phi}+\frac{\omega(\phi)}{\phi} H_{\phi}^{2}-3 H_{a} H_{\phi}, \\
\ddot{\phi}+3 H_{a} \dot{\phi} & +\frac{1}{2} \phi R-\frac{1}{2} \frac{d V}{d \phi}+\frac{1}{2} \dot{\phi}^{2}\left(\frac{\omega^{\prime}(\phi)}{\omega(\phi)}-\frac{1}{\phi}\right)=0,
\end{aligned}
$$

where $H_{a}=\dot{a} / a, H_{b}=\dot{b} / b$ and $H_{\phi}=\dot{\phi} / \phi$ augmented by the energy conservation equation

$$
\dot{\rho}+3 H_{a}(\rho+p)+6 H_{b}(\rho+P)=0 .
$$

It is almost unfeasible to solve the above set of field equations even in the homogeneous and isotropic FRW background geometry as these are only a pair of independent field equations, with at least five field variables, in addition to the density and pressure. A possible and realistic solution requires at least three additional assumptions to obtain exact analytical solutions. However, it may be possible to find a fixed form for $f$ and $\omega$ which would satisfy all the field equations from early Universe through matter dominated era with different cosmological evolution of $\phi$. Accordingly, in our approach, we believe that scaling solutions enable the asymptotic behaviour and stability of the cosmological background to be determined. Moreover, they provide a framework for establishing the behaviour of more general cosmological solutions. In what follows, we will assume in the visible section the equation of state $p=(\gamma-1) \rho, \gamma$ is a constant. Besides, we assume for simplicity that the scaling laws behavior: $\omega(\phi)=\omega_{0} \phi^{m}, V(\phi)=V_{0} \phi^{n}, a(t)=a_{0} t^{q}$, $b(t)=b_{0} t^{r}$ and $\phi(t)=\phi_{0} t^{p} \cdot p, q, m, n$ and $r$ are real constants and $\omega_{0}, V_{0}, a_{0}, b_{0}$ and $\phi_{0}$ are the values of the parameters at the present epoch assumed equal to unity for mathematical simplicity. The natural way of getting from $9+1$ a $3+1$ dimensional universe is to compactify the six spatial extradimensions by hand. For this motive, we make the hypothesis that $b(t)=(1 / a(t))^{N}, N \in \mathbb{R}^{+}$. This gives the simple relation $H_{b}=-N H_{a}$. [26] Then equations (5), (6) and (3) reduce straightforwardly to:

$$
\begin{aligned}
& 3 q t^{-2}(q(N-1)(5 N-1)+p) \\
& =t^{p n}+8 \pi \rho t^{-p}+p^{2} t^{p(m-1)-2}-36 C q N \\
& \times(5 N(N-1)(N-3)-2) q^{3} t^{-4}, \\
& \dot{\rho}+3 \gamma \rho \frac{q}{t}+6 \frac{r}{t}(\rho+P)=0, \\
& R=n t^{p n-2 p}-2\left(p-1+3 q+\frac{1}{2} p(m-1)\right) p t^{-2} .
\end{aligned}
$$

We already know that the behaviour of the Ricci scalar is dictated by the metric, nevertheless, it is obvious from equation (10) that, if for instance, we set $p(n-2)=-2$, a consistent solution is obtained if, for instance, the scalar curvature varies like $R=\xi t^{-2}, \xi \in \mathbb{R}$, i.e. $R \rightarrow 0$ at late time as required by inflation theory. Accordingly, equation (8) takes the special form:

$$
\begin{aligned}
& 3 q t^{-2}(q(N-1)(5 N-1)+p) \\
& =t^{2 p-2}+8 \pi \rho t^{-p}+p^{2} t^{p(m-1)-2}-36 C N \\
& \times(5 N(N-1)(N-3)-2) q^{4} t^{-4} .
\end{aligned}
$$

We are concerned on the involvement of the GB invariant curvature term to the dynamical equation of motion as GB gravity can lead to late-time accelerated expansion of the universe. For this, we select the consistent solution which is equivalent to the statement "mass without mass" [26,28]:

$$
\begin{aligned}
& \rho(t)=\frac{9 C N}{2 \pi}(5 N(N-1)(N-3)-2) q^{4} t^{p-4}-\frac{1}{8 \pi} t^{3 p-2} \\
& =-\frac{1}{8 \pi} t^{3 p-2} \\
& \times\left(-36 C N(5 N(N-1)(N-3)-2) q^{4} t^{-2-2 p}+1\right), \\
& =\frac{9 C N}{2 \pi}(5 N(N-1)(N-3)-2) q^{p} H_{a}^{4-p}-\frac{1}{8 \pi} q^{3 p-2} H_{a}^{2-3 p}, \\
& =-\frac{1}{8 \pi} q^{3 p-2} H_{a}^{2-3 p} \\
& \times\left(-36 C N(5 N(N-1)(N-3)-2) q^{2-2 p} H_{a}^{3 p-2} H_{a}^{4-p}+1\right) .
\end{aligned}
$$

Obviously, at the critical time $t_{c}=[1 / 36 C N(5 N(N-1)(N-3)-2)]^{-1 / 2(1+p)}$, the matter 
energy density vanishes with time. However, the results of large-scale structure surveys and results of measurements of masses of galaxies give best fit for density parameter for matter $\Omega_{m, 0}=0.3$. Accordingly, we would like to avoid for instance such an event and we choose $p=-1$ for which

$$
\begin{aligned}
& \rho(t)=-\left(-\frac{9 C N}{2 \pi}(5 N(N-1)(N-3)-2) q^{4}+\frac{1}{8 \pi}\right) t^{-5}, \\
& =-\left(-\frac{9 C N}{2 \pi}(5 N(N-1)(N-3)-2) q^{-1}+\frac{1}{8 \pi} q^{-5}\right) H_{a}^{5} .
\end{aligned}
$$

Therefore, equation (11) is reduced to:

$$
3 q t^{-2}(q(N-1)(5 N-1)+p)=p^{2} t^{-(m-1)-2},
$$

from which a consistent and realistic relation is obtained, if for instance, $m=1$ with $n=4$ from the constraint $p(n-$ $2)=-2$. The scalar field, the coupling function and the scalar potential evolve respectively like $\phi(t) \propto t^{-1}, \omega(\phi) \propto$ $\phi$ and $V(\phi) \propto \phi^{4}$ during the accelerated expansion of the universe. A positive matter energy density corresponds to $q^{4}>1 / 36 C N[5 N(N-1)(N-3)-2], N>0$. Notice that $N=1$ or $N=1 / 5$ give $3 q=p$. Furthermore, equation (10) gives $\xi=8-6 q$ and hence as long as $q<1.33, \xi>0$ and therefore the Ricci scalar curvature $R=(8-6 q) t^{-2}$ is positive or negative according to whether $q<1.33$ or $q>1.33$. We have, hence, a deviation from the standard cosmology which predicts a scalar curvature $R=0$. It is interesting to have an accelerated universe dominated by dark energy with scalar non-zero curvature. Equation (14) gives in its turn $3 q(q(N-1)(5 N-1)-1)=1$ from which one deduces straightforwardly:

$$
q=\frac{3 \pm \sqrt{9+12(N-1)(5 N-1)}}{6(N-1)(5 N-1)}
$$

An accelerated expansion may occur if, for instance, $q>1$. Hence, the positive and then negative signs of equation (15) are plotted in Figures 1 and 2 respectively:

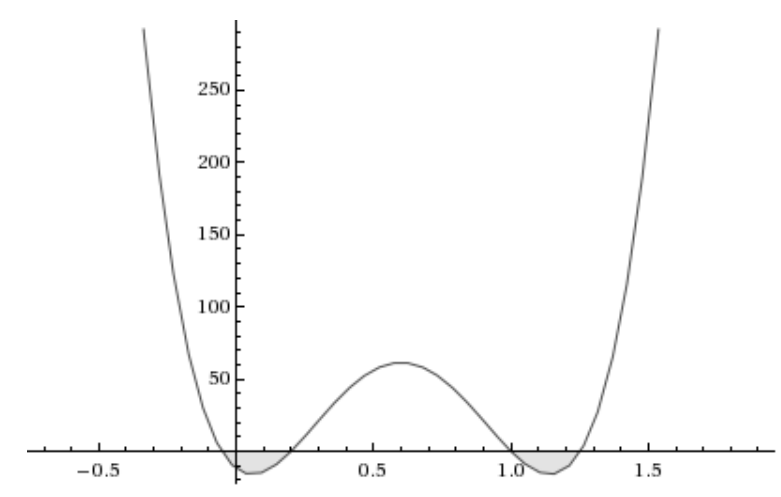

FIG. $1: q=\frac{3+\sqrt{9+12(N-1)(5 N-1)}}{6(N-1)(5 N-1)}>1$

Figure 1 gives the range

$$
1<N<\frac{1}{15}(9+4 \sqrt{6}),
$$

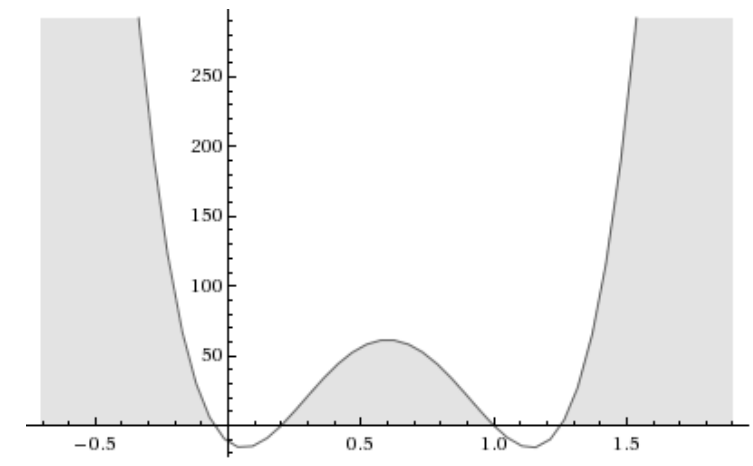

FIG. $2: q=\frac{3-\sqrt{9+12(N-1)(5 N-1)}}{6(N-1)(5 N-1)}>1$

$$
\frac{1}{15}(9-4 \sqrt{6})<N<\frac{1}{5}
$$

whereas Figure 2 gives the range:

$$
\begin{gathered}
N>\frac{1}{15}(9+4 \sqrt{6}), \\
\frac{1}{5}<N<1, \\
N<\frac{1}{15}(9-4 \sqrt{6}) .
\end{gathered}
$$

The constraint $q^{4}>1 / 36 C N[5 N(N-1)(N-3)-2], N>0$ gives now $C>-4 \cdot 10^{-3}$.

Two interesting features arise here:

1-The matter density obviously depends on the GaussBonnet coupling constant and behaves as $\rho \propto H_{a}^{5}$,

2-the scale factor parameter depends on the parameter $N$.

Furthermore, we assume that the pressure is isotropic including the extra-dimensions, i.e. $p=P$. Equation (9) is reduced to $\dot{\rho}+3(q+2 r) \gamma t^{-1} \rho=0$ and gives accordingly $p-4+3(q+2 r) \gamma=0$ or $3(q+2 r) \gamma=5$. It is noteworthy that the Hubble parameter $H(t) \equiv \dot{a} / a$ and the deceleration parameter $\bar{q}(t) \equiv-\ddot{a} a / \dot{a}^{2}$ are related by $\bar{q} \equiv-\ddot{a} / H \dot{a}=$ $-(q-1) / q$. In fact, the kinematic approach to cosmological tests provides direct confirmation of the present accelerating dynamics independently on the validity of General Relativity, as well as on the matter-energy content of the Universe. In this perspective, one may use a phenomenological two-linear parameter expansion for the decelerating parameter, i.e. $\bar{q}(z)=\bar{q}_{0}+\bar{q}_{1} z$ where $\bar{q}_{0}$ and $\bar{q}_{1}$ are arbitrary constants to be compared with the values constrained by the Union supernovae data. The recent astrophysical and observational data favor the recent acceleration $\bar{q}_{0}<0$ (Low Energy Limit) and past deceleration $\bar{q}_{1}>0$ (High Energy Limit) with high confidence level. To illustrate, we make use of the more recent results obtained by the Union $\mathrm{SNe}$ compilation as well as some of the results found in literature, in particular the recently extended dataset of distant supernovae observed with HST34, e.g. $\bar{q}_{0} \leq-0.25$ with $68 \%$ confidence level. We choose $N \approx 0.1$ from which one deduces easily: $q \approx 2.51$ for the positive sign and hence $\bar{q}_{0} \approx-0.6$. Hence 
from the constraint $3(q+2 r) \gamma=5$, we find $r=5 / 6 \gamma-1.255$. As naturally, we expect a contracting extra-dimensions during the accelerated expansion of the universe, then $r<0$ or $\gamma>0.66$ and the equation of state parameter is consequently $w=\gamma-1>-0.33$. While for $N \approx 1.05$, we find $q \approx 5$ and $\bar{q}_{0} \approx-0.8$. Accordingly, $r=5 / 6 \gamma-2.5$ and hence $w=\gamma-1>-2 / 3$. Both illustrations correspond to dark energy dominance. It is noteworthy that cosmologies with $w<-1$ have some potentially serious problems. One of the main serious problem concerns the fact that the speed of the energy flow may surpass the speed of light allowing closed causal loops. An additional problem is related to the Hamiltonian which can be unbounded below depending on the cosmological system, but mainly for phantom matter with a negative kinetic energy. Such a system has a negatively infinite ground state, therefore no stable vacuum solution exists [35]. Other problem is related to Big Rip, i.e. the universe expansion would accelerate at such a speedy rate that the event horizon would ultimately minimize to the point that all bound systems are ripped apart, as no particle can remain causally connected to any other particle.

The effective gravitational constant in the theory is here $G_{10} \propto \phi^{-1} \propto t$. The present day variation of the gravitational constant is $\left|\dot{G}_{10} / G_{10}\right|=2 H_{0} / q$. Notice that form $N \approx 1.05, q \approx 5$ and hence $\left|\dot{G}_{10} / G_{10}\right| \approx 0.4 H_{0}$ with $H_{0}=$ $80 \pm 7 \mathrm{~km}^{-1} \mathrm{Mpc}^{-1}$ in accordance with recent astronomical data [36]. It is noteworthy that over the years there have been many experiments performed that place upper limits on the time variability of the effective gravitational coupling constant. In general, it was recently observed that for late times, a modified cosmology with varying gravitational coupling constant is in accordance with the observed values of the cosmological parameters. [37]

In summary, if we admit the superstring theory as a true and promising quantum field theory of gravity unifying gravity with all matter interactions, the GB invariant term is the only grouping of curvature-squared interactions for which the low-energy effective action is ghost-free. In the present work our main aim was to explore the novel cosmological features resulting from the presence of the Gauss-Bonnet curvature term in 10-dimensional modified gravity with nonminimal coupling. We ignored the presence of the dilaton field and the Yang-Mills field strength, therefore our framework is different from the multidimensional scenarios discussed by Kripfganz-Perlt [38] and Lorentz-Petzold. [39] However, most of the previous works have been devoted to vacuum solutions. In order that the equivalence principle is satisfied, we have identified the scalar field as a run-away modulus without direct matter couplings though gravitational dynamics are modified due to the presence of modulus-dependent loop corrections. We showed that such coupling can trigger the accelerated expansion of the universe without the presence of phantom energy field after a scaling matter era. Besides investigating the late-time cosmological evolution of such models, we also derive quantitative constraints on the scenario of Gauss-Bonnet coupling function. We have choose scaling solutions as we strongly believe that scaling solutions during the dynamical evolution of the universe play a crucial role as they serve to better understand many extended properties. Moreover, these scaling solutions can lead in reality to a viable late-time cosmology with the accelerated expansion starting earlier $(z>1)$ than in common (uncoupled) dark energy cosmological models but still consistent with the recent SNeIa supernovae astrophysical data. The GB coupling parameter is subdominant to the Einstein term and decays like $f(t) \propto t^{-1}$. Since the Einstein terms go also as $R \propto t^{-2}$, the effects of the Gauss-Bonnet correction term on the late-time universe is small, but it still plays an important role in the cosmic evolution. In other words, the linear curvature term therefore dominates over the contribution of the quadratic term and all other higher order terms if curvature is non-minimally coupled to the scalar field. The factor $f(\phi) R$ varies like $f(\phi) H_{a}^{2}$ as $R \propto H_{a}^{2}$ for the factor $C G(R) \propto t^{-4}=H_{a}^{4}$ to remain subdominant as it is expected through the evolution of the universe. The term $f(\phi) R$ plays here a leading role with regard to the effect of the Gauss-Bonnet term on the evolution of the universe.

We have obtained a late time accelerated expansion of the universe without crossing the phantom divide line, i.e. without the presence of phantom field of any kind and independent of the signature of the GB coupling parameter. There exist other specific scenarios of dark energy with striking features capable of explaining the present accelerated expansion of the universe $[40,41,42,43,44,45]$. The simple model described in this paper belongs to the same sort but holds some new features discussed above. This model needs a firmer theoretical supporting which might come from modified gravity theories. Other interesting consequences may be revealed in particular the luminosity distance expression and statistical analysis of the data obtained; however, it is a primitive model and work in this direction is under progress. This only shows that such investigations may be useful for a future study.

\section{Acknowledgments:}

The author would like to thanks the anonymous referees for their useful comments and valuable suggestions.
[1] A.G. Riess et al., (1998) Astron. J. 116, 1009.

[2] S. Perlmutter et al., (1999) Astrophys. J. 517, 565.

[3] B.R. Schmidt et al., (1998) Astrophys. J. 50746.

[4] P.J. Steinhardt, L. Wang and I. Zlatev, (1999) Phys. Rev. D 59 123504.

[5] M. Persic, P. Salucci and F. Stel, (1996) Mon. Not. Roy. Astron. Soc. 281, 27.

[6] M. Carmeli and T. Kuzmenko, (2002) Int. J. Theor. Phys. 41, 131.
[7] J.S. Alcaniz, (2004) Phys. Rev. D69, 083521.

[8] P. de Bernardis, (2000) Nature, 377, 600; see also Sahni V and Starobinsky A astro-ph/0610026.

[9] P.J.E. Peebles and Ratra B, (2003) Rev. Mod. Phys. 75, 559.

[10] Ph Brax and J. Martin (1999) Phys. Lett. B468, 45.

[11] C. Armendariz-Picon, V. Mukhanov and P.J. Steinhardt (2001) Phys. Rev. D63, 103510.

[12] J.C. Fabris, S.V.B. Concalves and de R. Sa Ribeiro, (2006) Gen. Rel. Grav. 38, 495. 
[13] A. Yu Kamenshchik, V. Moschella and V. Pasquier, (2001) Phys. Lett. B511, 265.

[14] J.C. Fabris, S.V.B. Concalves and P.E. de Souza, (2002) Gen. Rel. Grav. 34, 53.

[15] N. Bilic, G.B. Tupper and R.D. Viollier (2002) Phys. Lett. B535, 17.

[16] N.C. Bento, O. Bertolami and A.A. Sen, (2002) Phys. Rev. D66, 043507.

[17] A.B. Batista, J.C. Fabris and de R. Sa Ribeiro (2001) Gen. Rel. Grav. 33, 1237.

[18] A.A. Sen, S. Sen and S. Sethi, (2001) Phys. Rev._D63, 10750.

[19] S. Sen and T.R. Seshadri, (2003) Int. J. Mod. Phys. D12, 445 and references therein.

[20] M.C. Bento and O. Bertolami, (1996) Phys. Lett. B368, 198 and references therein.

[21] W-Y P. Hwang and J. Tsai, (2004) Nucl. Phys. B700, 313.

[22] A.A. Sen, (2000) PRAMANA J. Phys. 55, No. 3, 369.

[23] A.R. El-Nabulsi, (2006) Chin. Phys. Lett. 23, No 51124.

[24] A.R. El-Nabulsi, (2005) Phys. Lett. B619, No 1-2 26.

[25] A.R. El-Nabulsi, (2008) Chin. Phys. Lett. 25, No. 8, 2785.

[26] A.R. El-Nabulsi, (2009) Int. J. Mod. Phys. D18, 15, 691.

[27] A.R. El-Nabulsi, (2008) Mod. Phys. Lett. A23, No. 6, 401.

[28] A.R. El-Nabulsi, (2009) Int. J. Mod. Phys. D18, 2, 289.

[29] A.R. El-Nabulsi, (2009) Comm. Theor. Phys. (in press).

[30] A.K. Sanyal arXiv:0910.2385 (astro-ph.CO).

[31] A.L. Maroto and I.L. Shapiro (1997) Phys. Lett. B414, 34; Bamba K, Guo Z K and Ohta N (2007) Prog. Theor. Phys. 18,
879.

[32] D. Naresh in Proceedings of 12th Regional Conference on Mathematical Physics, Islamabad, March, 27 - April, 1, 2006 (hep-th/0509126).

[33] S. Kanno, D. Langlois, M. Sasaki and J. Soda, (2007) Prog Theor. Phys. 118, 701.

[34] E.V. Pitjeva, (2005) Astron. Lett. 31, 340.

[35] G.M. Hossein, (2005) Class. Quantum Grav. 22, 2653; see also Leith B M (2007), Scalar fields and alternatives in cosmology and black holes, $\mathrm{PhD}$ Thesis, University of Canterbury.

[36] J.V. Cunha (2009) Phys. Rev. D79, 047301.

[37] J.C. Carvalho, J.A.S. Lima and I. Waga, (1992) Phys. Rev D46, 2404; Alcaniz J S and Maia J M F (2003) Phys. Rev. D67, 043502.

[38] J. Kripfganz and H. Perlt, (1987) Acta Phys. Pol. B18, 11, 977.

[39] D. Lorentz-Petzold, (1988) Mod. Phys. Lett. A3, 827.

[40] A.R. El-Nabulsi (2009) Astrophys. Space Scie. 325, 2, 277.

[41] A.R. El-Nabulsi, (2010) Astrophys. Space Scie. 325, 2, 149.

[42] A.R. El-Nabulsi (2009) Astrophys. Space Scie. 324, 1, 71.

[43] A.R. El-Nabulsi, Astrophys. Space Scie. DOI 10.1007/s10509009-0259-4 (in press).

[44] A.R. El-Nabulsi, Astrophys. Space Scie. DOI 10.1007/s10509009-0262-9 (in press).

[45] B. Giorgini and R. Kerner (2008) Classical and Quantum Gravity 5, 339. 